International Journal of Pure and Applied Mathematical Sciences.

ISSN 0972-9828 Volume 9, Number 2 (2016), pp. 145-164

(C) Research India Publications

https://dx.doi.org/10.37622/IJPAMS/9.2.2016.145-164

\title{
An Integrated Genetic Algorithm with Clone Operator
}

\author{
G. S. Biju ${ }^{1}$ and A. K. Anilkumar ${ }^{2}$ \\ ${ }^{1}$ Department of Mathematics, College of Engineering, \\ Thiruvananthapuram, Kerala-695016, India. \\ ${ }^{2}$ Vikram Sarabhai Space Centre, Indian Space Research Organisation, \\ Thiruvananthapuram, Kerala, India.
}

\begin{abstract}
Genetic algorithm has got much interest and application as an optimization tool. In this paper we propose a new operator based on the concept of cloning. The proposed new operator imitates the biological cloning process. Based on a simple genetic algorithm and new operator, we propose an integrated genetic algorithm. The new algorithm is validated with standard test cases and is shown that the new operator helps in getting faster convergence and global optima.
\end{abstract}

Keywords: Optimization, Evolutionary Algorithm, Genetic Algorithm, Clone operator

\section{INTRODUCTION}

Genetic algorithm is a partial search algorithm designed by observing and mimicking the intelligent mechanism behind biological evolutions such as reproduction, recombination, mutation and selection, which may be used to obtain sufficiently good approximations to various classes of optimization problems. To find the optimal solution of a problem, an environment is created in which potential solutions can evolve naturally, with the parameters of the problem serving to control and shape the environment. The quality of the solutions so obtained are checked by one or more fitness functions at every stage. However, the evaluation of the fitness function often renders the problem computationally complex, and to overcome this, various approximations to fitness functions are generally used. Very complex optimization problems are sometimes solved by surprisingly simple genetic algorithms. However, the complexity of the problem has in general no direct link with the complexity of the 
algorithm. A major advantage of a genetic algorithm is its generality, as it ideally does not make any assumptions about the fitness landscape. Genetic algorithms have been successfully applied in solving optimization problems of various types in fields as diverse as engineering, art, biology, economics, marketing, genetics, operations research, robotics, social sciences, physics, politics and chemistry.

The basic principles of GAs were introduced, for the first time, by John Henry Holland [1] for solving practical optimization problems and are well documented [210]. The aim of a GA is to achieve better results through selection, crossover and mutation. The success of any GA depends on the design of its search operators as well as their appropriate integration. There is a lot of literature to improve the effectiveness of the GA operators. There are various approaches [3,11,2,12-17] suggested to select the parent's string which may vary depending on the difficulty level of the problems. In a GA, maintaining population diversity is very important to reach to the global optimum [18]. It is necessary to select the best solution of the current generation to direct a GA to reach to the global optimum. Goldberg and Deb [19] compare four different selection schemes: proportionate selection, fitness ranking, tournament selection and steady state selection. They conclude that by suitable adjustment of parameters, all these schemes can be made to give similar performances, so there is no absolute best method. Many different crossover algorithms are devised [20,17, 21]. DeJong [22] investigated the effectiveness of multiple-point crossover. Besides, the initial population plays an important role in solving a problem by GA [23-26]. Genetic algorithms have been successfully used in numerical function optimization [22], image processing [3], combinatorial optimization [27], machine learning [2830], data mining, information retrieval and grammar induction.

A basic genetic algorithm comprises of three genetic operators, namely, selection, crossover and mutation. Reproduction(selection) is usually the first operator applied on a population. Reproduction selects good strings in a population and forms a mating pool. There exists a number of reproduction operators in GA literature, but the essential idea in all of them is that the above average strings are picked from the current population and their multiple copies are inserted in the mating pool in a probabilistic manner.

In the crossover operator, new strings are created by exchanging information among strings of the mating pool. Many crossover operators exist in the GA literature. In most crossover operators, two strings are picked from the mating pool at random and some portions of the strings are exchanged between the strings. The two strings participating in the crossover operation are known as parent strings and the resulting strings are known as offspring strings. In practice, all parents in the mating pool are not selected for crossover operation so that some of the good strings may be preserved. This is achieved by selecting a fixed percentage of parents from the mating pool and it is known as the crossover probability. 
Mutation is applied to each child individually after crossover. This operator randomly flips or alters one or more bit values at randomly selected locations in a chromosome with a small probability.

The overall GA process steps are summarized as follows:

STEP 1 Choose coding to represent problem parameters: a selection operator, a crossover operator, and mutation operator.

STEP 2 Choose initial population size, length of a string, probabilities of cross over and mutation, search domain of the variables, termination criteria or maximum number of iteration.

STEP 3 Set $\mathrm{T}=0$. Generate initial population from the search domains randomly.

STEP $4 \quad$ Evaluate each string of the population for fitness.

STEP 5 If termination criteria is satisfied, or T $>$ Tmax, then STOP.

STEP 6 Perform selection on the population.

STEP 7 Perform crossover on random pairs of strings.

STEP 8 Perform mutation on every string.

STEP 9 Evaluate the strings of the new population.

STEP $10 \quad$ Set $\mathrm{T}=\mathrm{T}+1$, go to step 4 .

In this paper, firstly, we propose a new operator based on the concept of cloning. Section 2 gives a description of the new operator and its illustration; and we propose an evolutionary algorithm which integrates the new operator with the other GA operators. In this new GA, the selection process can be absent as well. Thereafter, in section 3, we describe the proposed CGA algorithm in detail. The computational results of test problems are shown in section 4. Finally, section 5 summarizes the concluding remarks.

\section{CLONE OPERATOR}

We introduce a new operator called Clone Operator. Cloning of genes in the elitist set is fundamental to this approach. The elitist member(or few members) of a generation is cloned to get a member of the next generation. Cloning is carried out with a clone probability. The genes which are not cloned based on probability are mutated $(0$ $\rightarrow 1,1 \rightarrow 0$ ). It is expected that the new member will have better qualities of the parent member. As an example, consider the string

\begin{tabular}{|l|l|l|l|l|l|l|l|l|l|}
\hline 1 & 0 & 1 & 1 & 1 & 0 & 1 & 0 & 0 & 1 \\
\hline
\end{tabular}

On applying the operator, each bit may undergo a cloning with a certain probability. If the cloning probability is 0.8 , two genes of the string will be mutated randomly and we might get, after cloning, the string-

\begin{tabular}{|l|l|l|l|l|l|l|l|l|l|}
\hline 1 & 0 & 1 & 0 & 1 & 0 & 1 & 1 & 0 & 1 \\
\hline
\end{tabular}

in which two genes, viz. the fourth and the eighth, are changed while all the others are cloned. Initial population of the GA with cloning can start either with a random population or with a population with all of its members having genes 1 or 0 . One 
generation to next generation, in this new proposed GA, will not contain the saplings generated by GA operators like crossover and mutation. New generation will be containing only the elitist member from the previous generation on which clone is operated.

\section{Demonstration of cloning from generation to generation}

Consider the problem,

Minimize $\mathrm{f}(\mathrm{x}, \mathrm{y})=(\mathrm{x}-1)^{2}+(\mathrm{y}-2)^{2}$ where $-5 \leq x, y \leq 5$

This function has global minimum value 10 at $(1,2)$. Let the initial population consists of 10 strings of elites

1111111111111111. Let 0.75 be the cloning probability. The first two generation are given as follows;

Initial population: Elite 1111111111111111

$\mathrm{x}=5, \mathrm{y}=5$, function minimum $=35$.

\begin{tabular}{|c|c|c|c|}
\hline $\begin{array}{l}\text { Population } \\
\text { after cloning }\end{array}$ & $\mathrm{x}$ & $\mathrm{y}$ & $\begin{array}{c}\text { Function } \\
\text { value }\end{array}$ \\
\hline 1011101110111011 & 2.3333 & 2.3333 & 11.8889 \\
\hline 0110111101110111 & -0.6471 & -0.3333 & 18.1572 \\
\hline 1101101111111001 & 3.5882 & 4.7647 & 24.3426 \\
\hline 1011010111011111 & 2.0980 & 3.7451 & 14.2511 \\
\hline 0011101110111111 & -2.6863 & 2.4902 & 23.8289 \\
\hline 1111011101101110 & 4.6863 & -0.6863 & 30.8047 \\
\hline 1011111110110101 & 2.4902 & 2.0980 & 12.2303 \\
\hline 1110110111001111 & 4.2941 & 3.1176 & 22.1003 \\
\hline 0011111111111100 & -2.5294 & 4.8824 & 30.7647 \\
\hline 1011111111010111 & 2.4902 & 3.4314 & 14.2695 \\
\hline
\end{tabular}

Elite : 1011101110111011

$\mathrm{x}=2.3333, \mathrm{y}=2.3333$, function minimum $=11.8889$.

The second generation consists of 10 strings of elites 1011101110111011 . 


\begin{tabular}{|c|c|c|c|}
\hline $\begin{array}{l}\text { Population } \\
\text { after cloning }\end{array}$ & $\mathrm{x}$ & $\mathrm{y}$ & $\begin{array}{c}\text { Function } \\
\text { value }\end{array}$ \\
\hline 10001110010110111 & 1.0392 & & 17.8635 \\
\hline 0111101110101010 & -0.1765 & 1.6667 & 11.4952 \\
\hline 1010111110101010 & 18607 & 1.6667 & 10.8554 \\
\hline 1010101010101111 & 1.6667 & 1.8627 & 10.4633 \\
\hline 1001101010101111 & & & \\
\hline 1110101110111000 & 4.2157 & & 20.3872 \\
\hline 1100101110110011 & 2.9608 & 2.0196 & 13.8451 \\
\hline $\begin{array}{llllllll}1 & 0 & 1 & 1 & 0 & 0 & 1 & 1\end{array}$ & & & 13.4391 \\
\hline 0011101100101111 & -2.6863 & -3.1569 & 50.1819 \\
\hline 1010101101111010 & 1.7059 & -0.2157 & 15.4075 \\
\hline
\end{tabular}

Elite : 1001101011001111

$\mathrm{x}=1.0392, \mathrm{y}=1.8627$, function minimum $=10.0204$

We see that, using the new clone operator, we get closer to the global minimum just after two generations. This motivates us to develop a simple evolutionary algorithm, Cloned Evolutionary Algorithm(CEA), which is described as follows:

STEP 1 Choose coding to represent problem parameters.

STEP 2 Input the initial base population size, string length, probability for mutation on the initial population, search domain of the variables, termination criteria or maximum number of iteration as Tmax.

STEP 3 Set $\mathrm{T}=0$. Generate initial base population.

STEP 4 Perform cloning on the base population with specified probability.

STEP 5 Find the best value and the best solution.

STEP 6 Create a new population by replacing all with the best member.

STEP 7 Evaluate each string of the population for fitness.

STEP 8 If termination criteria is satisfied or T $>$ Tmax, then STOP.

STEP $9 \quad$ Set $\mathrm{T}=\mathrm{T}+1$, go to step 5 . 
The flowchart for CEA is shown in Figure 1.

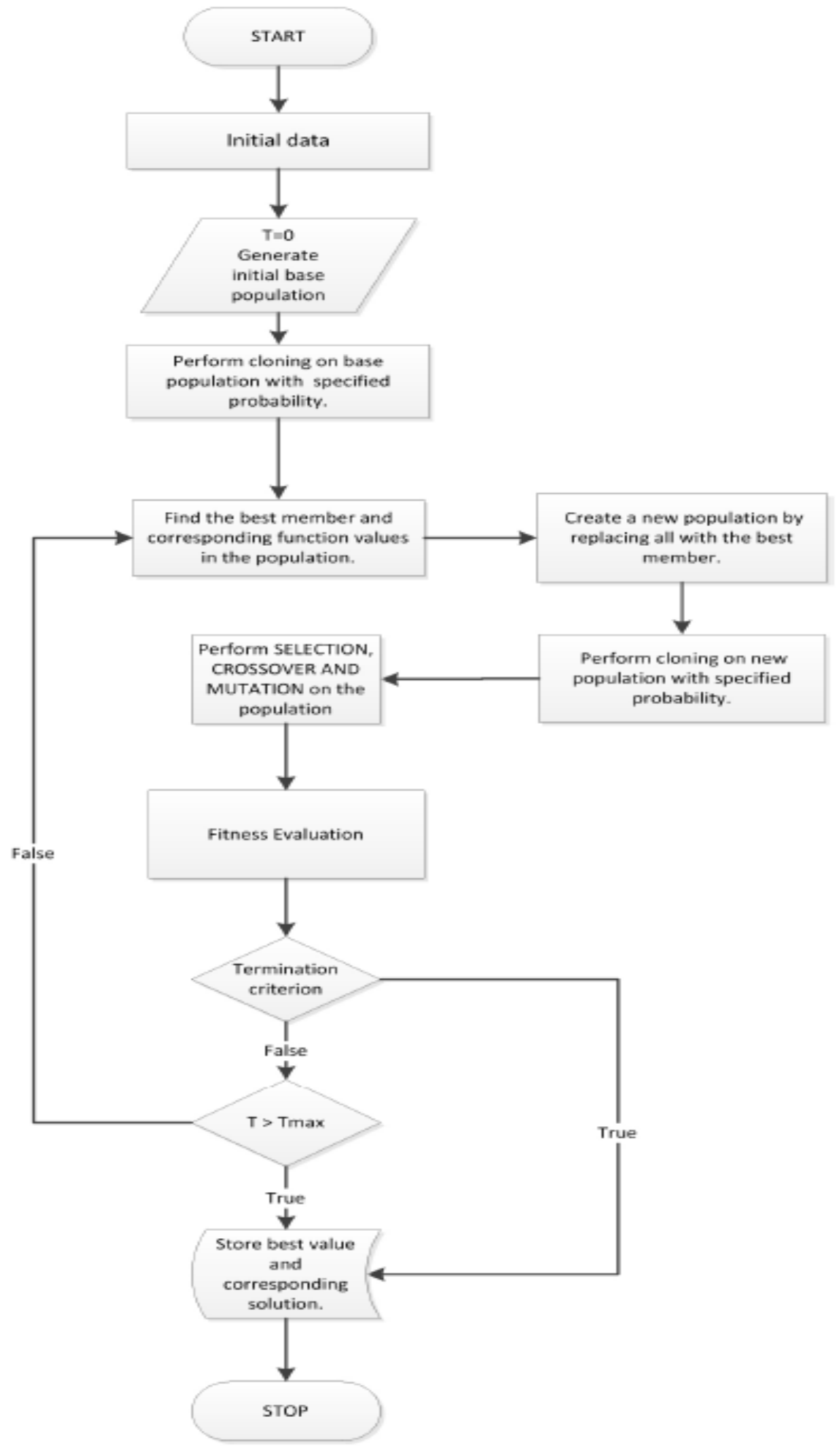

Figure 1: Flowchart for CEA 


\section{AN INTEGRATED GENETIC ALGORITHM}

In order to increase the accuracy, we make some modification in CEA. After creating a new population using clone operator, apply the GA operators reproduction, crossover and mutation on the new population. This leads to an innovative and improved evolutionary algorithm: Cloned Genetic Algorithm(CGA).

The step by step procedure of CGA can be summarized as follows:

STEP 1 Choose coding to represent problem parameters, a selection operator, a crossover operator, and mutation operator.

STEP 2 Input the initial base population size, string length, probability for cloning, search domain of the variables, probabilities of cross over, mutation probability, termination criteria or maximum number of iteration as Tmax.

STEP 3 Set $\mathrm{T}=0$. Generate initial base population.

STEP 4 Perform cloning on the base population with specified probability.

STEP 5 Evaluate each string of the population for fitness.

STEP 6 Perform selection on the population. 


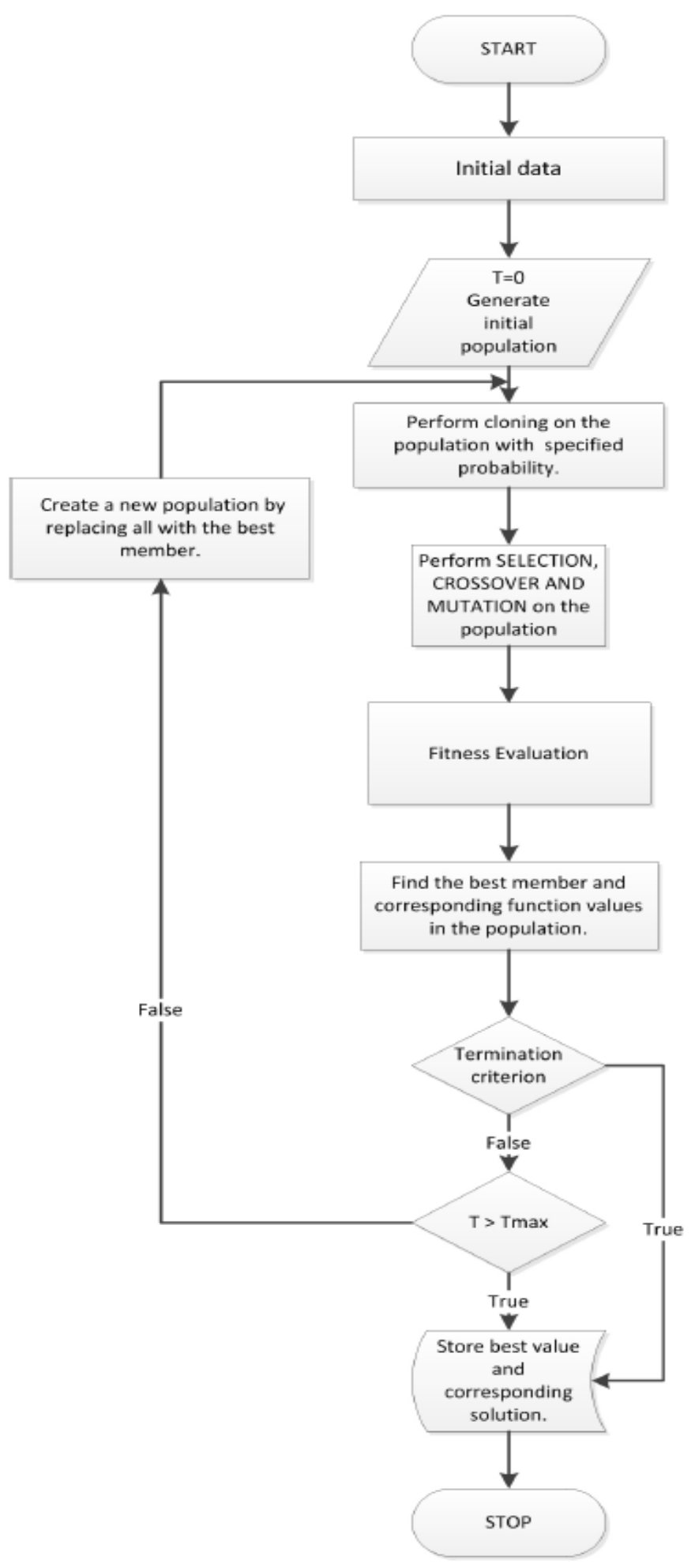

Figure 2: Flowchart for CGA 
STEP 7 Perform crossover on random pairs of strings in the population.

STEP 8 Perform mutation on every string in the population.

STEP 9 Evaluate each string of the population for fitness.

STEP 10 Find the best member and corresponding function values in the population.

STEP 11 If Termination Criteria is satisfied, or T $>$ Tmax, THEN jump to STEP-13. ELSE Jump to STEP 12

STEP 12 Set $\mathrm{T}=\mathrm{T}+1$. Create new population by replacing all with the best member.AND jump to STEP 4.

STEP 13 Store best value and corresponding solution. Then STOP.

The flowchart for CGA is shown in Fig. 2.

\section{CASE STUDIES}

We evaluate the performance of the proposed CGA and CEA on a set of 15 benchmark test functions (see Table 1 and Table 2) in the literature and 15 benchmark test functions in CEC05(see Table 3) with different complexity. For CEC05 benchmark problems, the problem definition files, codes are available at the web site address: http://www.ntu.edu.sg/home/EPNSugan. Experiments are conducted on the 2-D problems. The results of CGA and CEA on 2-D problems on population size = 100 are compared with simple GA. Parameters used in the implementation of CGA, CEA and GA are : Population Size $=100$, bit length for one variable $=16$, probability of clone $=$ random, probability of crossover $=0.80$, probability of mutation $=0.05$, maximum number of generations for convergence $=1000$ and alternative tolerance for convergence $=0.0000000001$.

We use the MATLAB to implement the algorithms. All the implementations were developed using a computer with processor Core 2 Quad processor of 64 bits that works to a frequency of $2.7 \mathrm{GHz}, 4 \mathrm{~GB}$ of RAM Memory and Windows XP Professional Version. In table 4 and table 5, we can find the experimental results. The table 4 and table 5 provides the results with 50 Monte-Carlo simulations with the changes in the random sequence. The results are provided in terms of statistical parameters such as average, the median, the best value, the worst value and standard deviation obtained for each test functions. The number of generations for convergence of the functions taken by the three methods is shown in table 6 and table 7 . The convergence maps of the algorithm on CGA, CEA and GA functions $\mathrm{g}_{1}$ to $\mathrm{g}_{5}$, functions $g_{6}$ to $g_{10}$, functions $g_{11}$ to $g{ }_{15}$, functions $f_{1}$ to $f_{5}$, functions $f_{6}$ to $f_{10}$, functions $f_{11}$ to $f_{15}$ are plotted in figures $3-8$ respectively.

The experimental analysis shows that the algorithm converges quickly to the optimal solution and thus exhibits a superior performance in comparison to GA algorithms. 

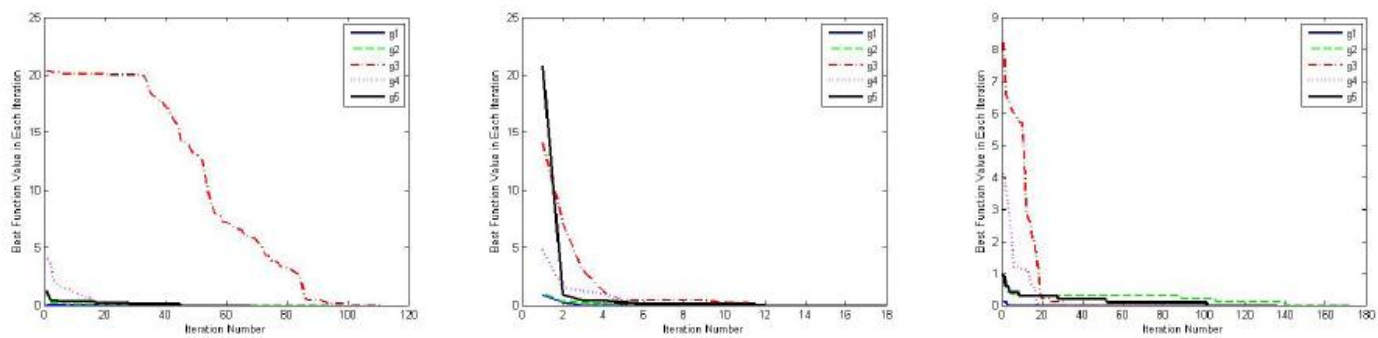

Figure 3: Convergence Graph for functions $g_{1}-g_{5}$ using CGA, CEA and GA algorithms. Left most plots are for CGA algorithm, middle plots are for CEA algorithm and right most plots are for GA algorithm.
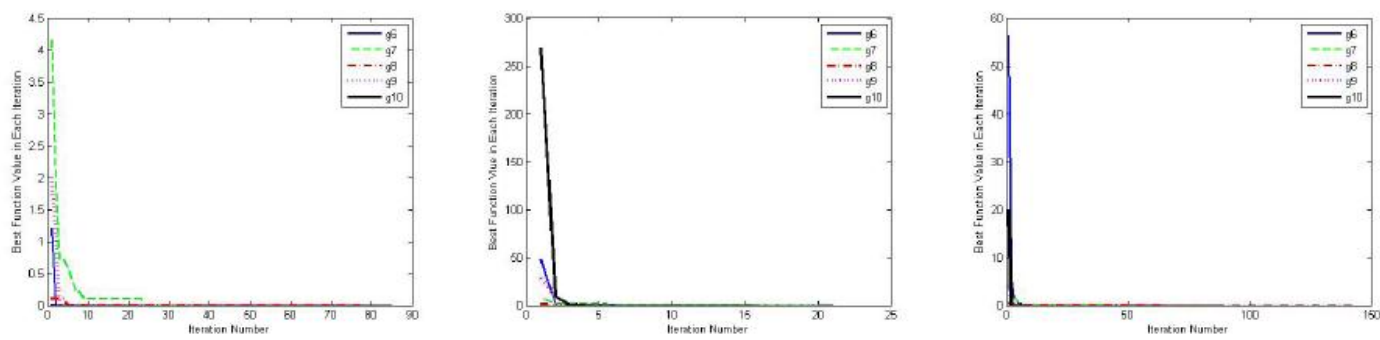

Figure 4: Convergence Graph for functions $\mathrm{g}_{6}-\mathrm{g}_{10}$ using CGA, CEA and GA algorithms. Left most plots are for CGA algorithm, middle plots are for CEA algorithm and right most plots are for GA algorithm.
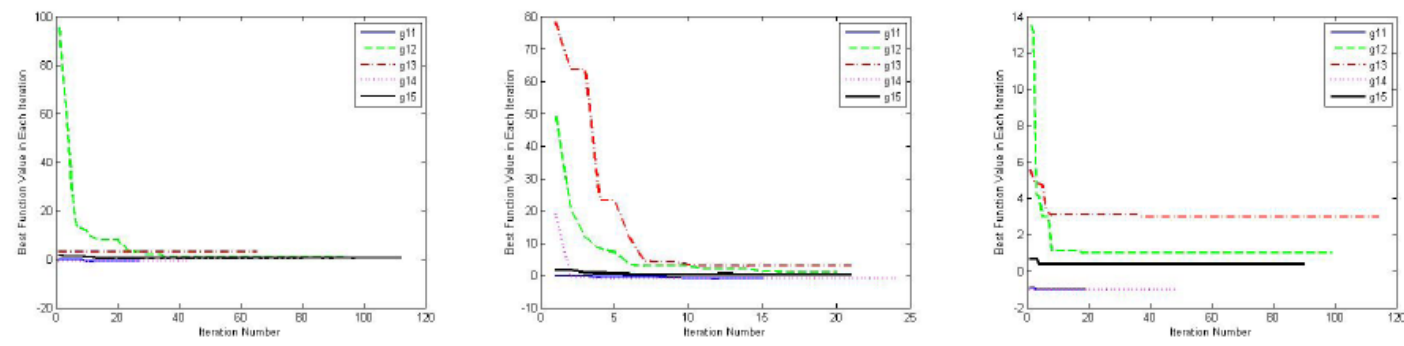

Figure 5: Convergence Graph for functions $g_{11}-g_{15}$ using CGA, CEA and GA algorithms. Left most plots are for CGA algorithm, middle plots are for CEA algorithm and right most plots are for GA algorithm. 

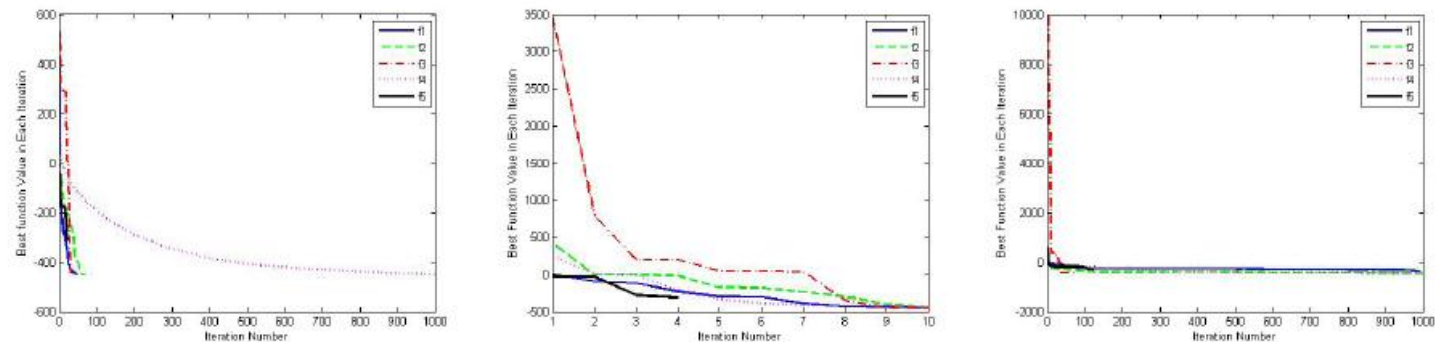

Figure 6: Convergence Graph for functions $f_{1}-f_{5}$ using CGA, CEA and GA algorithms. Left most plots are for CGA algorithm, middle plots are for CEA algorithm and right most plots are for GA algorithm.
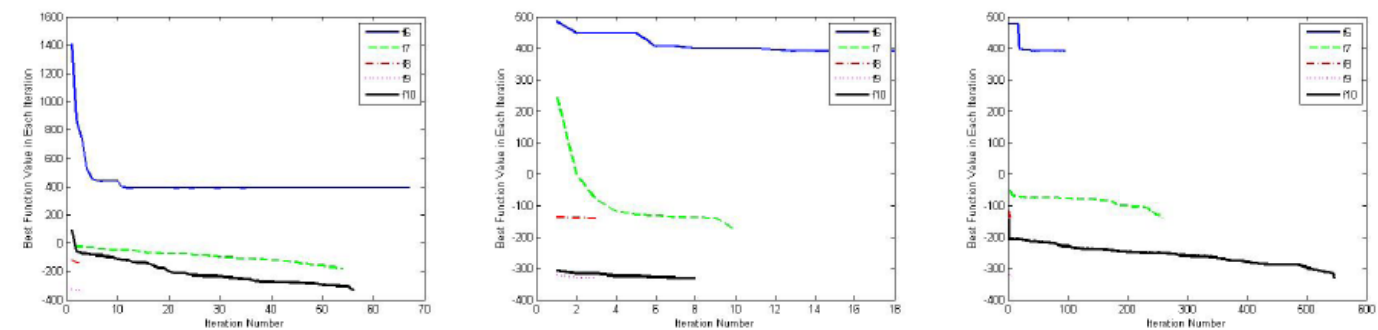

Figure 7: Convergence Graph for functions $f_{6}-f_{10}$ using CGA, CEA and GA algorithms. Left most plots are for CGA algorithm, middle plots are for CEA algorithm and right most plots are for GA algorithm.
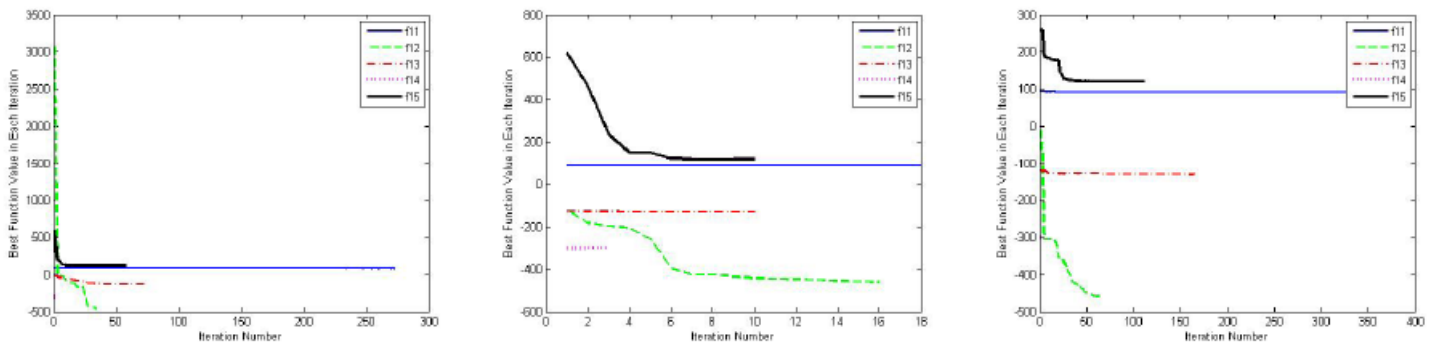

Figure 8: Convergence Graph for functions $f_{11}-f_{15}$ using CGA, CEA and GA algorithms. Left most plots are for CGA algorithm, middle plots are for CEA algorithm and right most plots are for GA algorithm. 
Table 1: Test functions $\mathrm{g}_{1}$ to $\mathrm{g}_{10}$

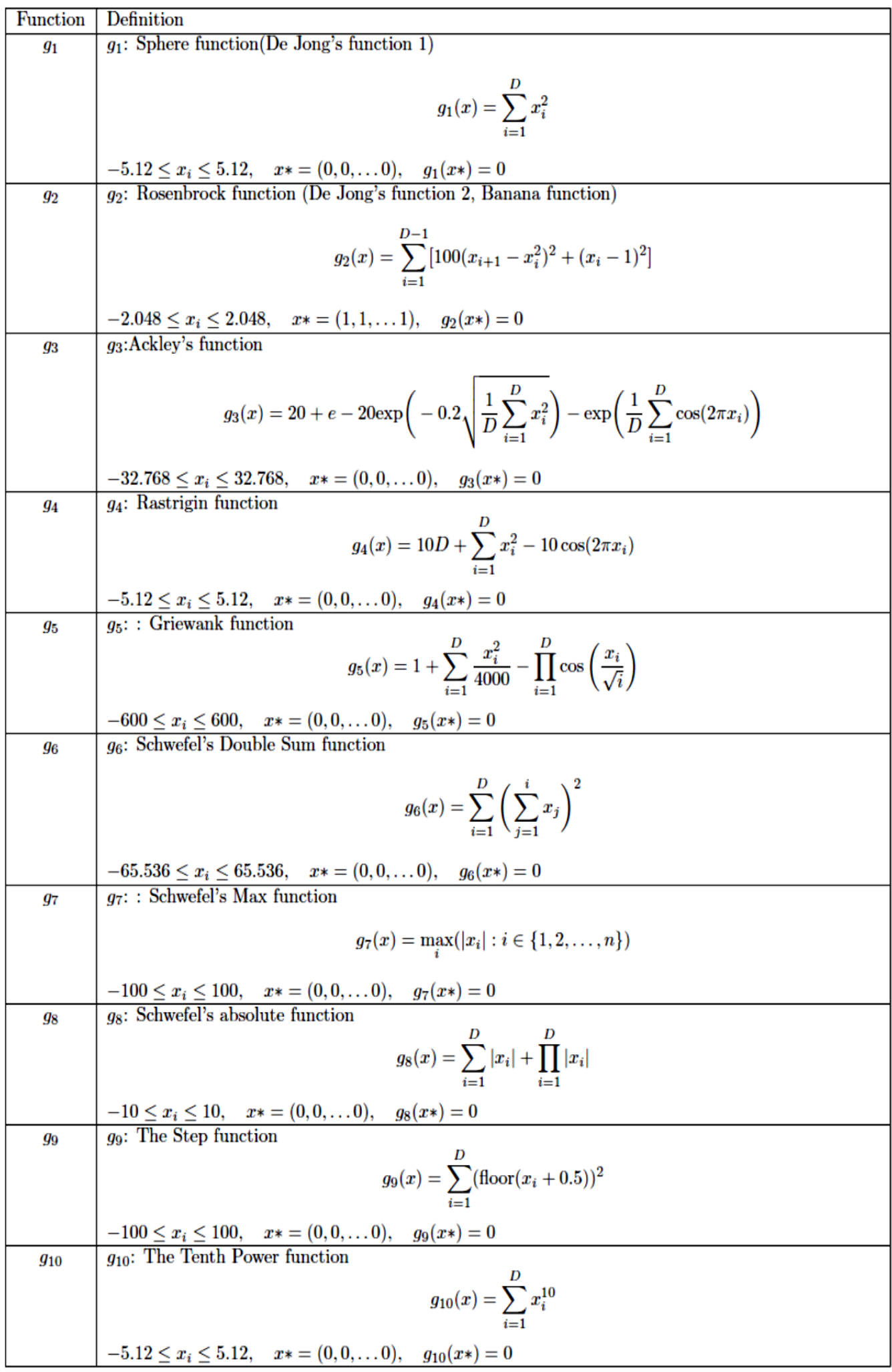


Table 2: Test functions $\mathrm{g}_{11}$ to $\mathrm{g}_{15}$

\begin{tabular}{|c|c|}
\hline Function & Definition \\
\hline$g_{11}$ & $\begin{array}{l}g_{11} \text { Easom function } \\
\qquad g_{11}(x)=-\cos \left(x_{1}\right) \cos \left(x_{2}\right) \exp \left(-\left(x_{1}-\pi\right)^{2}-\left(x_{2}-\pi\right)^{2}\right) \\
-100 \leq x_{i} \leq 100, \quad x *=(\pi, \pi), \quad g_{11}(x *)=-1\end{array}$ \\
\hline$g_{12}$ & 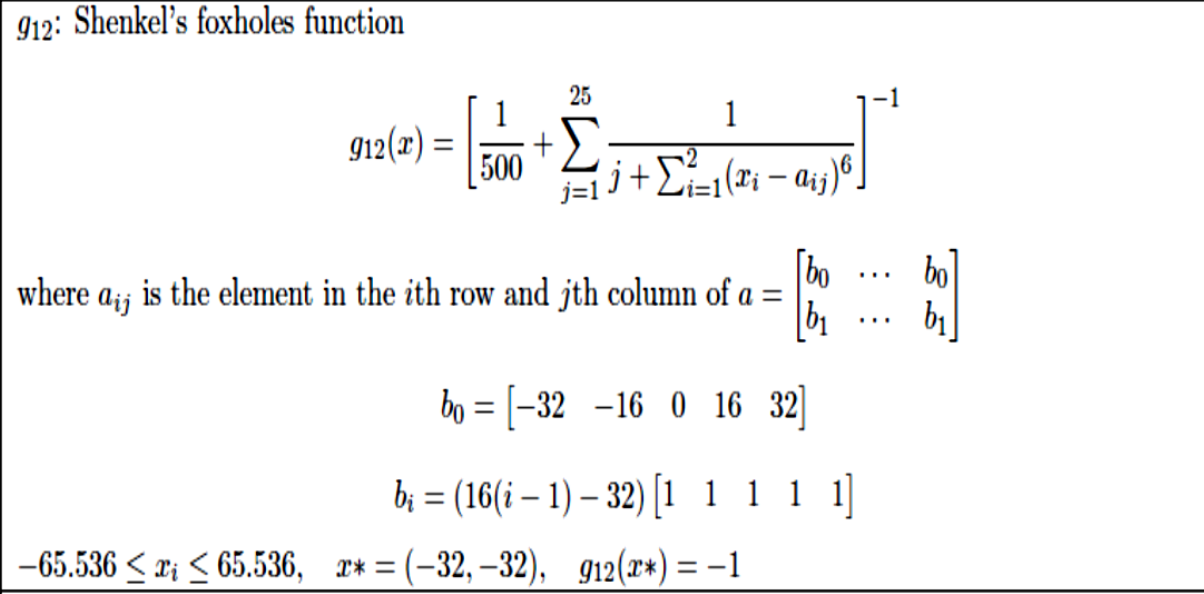 \\
\hline$g_{13}$ & $\begin{array}{l}g_{13} \text { : Goldstein-Price function } \\
\qquad \begin{array}{l}g_{13}(x)=\left[1+\left(x_{1}+x_{2}+1\right)^{2}\left(19-14 x_{1}+3 x_{1}^{2}-14 x_{2}+6 x_{1} x_{2}+3 x_{2}^{2}\right)\right] \\
\times\left[30+\left(2 x_{1}-3 x_{2}\right)^{2}\left(18-32 x_{1}+12 x_{1}^{2}+48 x_{2}-36 x_{1} x_{2}+27 x_{2}^{2}\right)\right] \\
-2 \leq x_{i} \leq 2, \quad x *=(0,-1), \quad g_{13}(x *)=3\end{array}\end{array}$ \\
\hline$g_{14}$ & $\begin{array}{l}g_{14} \text { : Six hump camel back function } \\
\qquad g_{14}(x)=\left[\left(4-2.1 x_{1}^{2}+x_{1}^{4 / 3}\right) x_{1}^{2}+x_{1} x_{2}+\left(-4+4 x_{2}^{2}\right) x_{2}^{2}\right. \\
-3 \leq x_{1} \leq 3,-2 \leq x_{2} \leq 2, \quad x *=(-0.0898,0.7126),(0.0898,-0.7126), \quad g_{14}(x *)=-1.0316\end{array}$ \\
\hline$g_{15}$ & $\begin{array}{l}g_{15} \text { : Branin's rcos function } \\
\qquad g_{15}(x)=\left(x_{2}-\frac{5.1}{4 \pi^{2}} x_{1}^{2}+\frac{5}{\pi} x_{1}-6\right)^{2}+\left(10-\frac{5}{4 \pi}\right) \cos x_{1}+10 \\
-5 \leq x_{1} \leq 10,0 \leq x_{2} \leq 15, \quad x *=(-\pi, 12.275),(\pi, 2.275),(9.42478,2.475) \quad g_{15}(x *)=0.397887\end{array}$ \\
\hline
\end{tabular}


Table 3: CEC 05 benchmark functions $\mathrm{f}_{1}$ to $\mathrm{f}_{15}$

\begin{tabular}{|c|l|c|c|}
\hline Function & Definition & $\mathrm{D}$ & $f_{\min }$ \\
\hline$f_{1}$ & Shifted Sphere Function(F1) & {$[-100,100]^{D}$} & -450 \\
$f_{2}$ & Shifted Schwefel's Problem 1.2(F2) & {$[-100,100]^{D}$} & -450 \\
$f_{3}$ & Shifted Rotated High Conditioned Elliptic Function(F3) & {$[-100,100]^{D}$} & -450 \\
$f_{4}$ & Shifted Schwefel's Problem 1.2 with Noise in Fitness(F4) & {$[-100,100]^{D}$} & -450 \\
$f_{5}$ & Schwefels Problem 2.6 with Global Optimum on Bounds(F5) & {$[-100,100]^{D}$} & -310 \\
$f_{6}$ & Shifted Rosenbrock's Function(F6) & {$[-100,100]^{D}$} & 390 \\
$f_{7}$ & Shifted Rotated Griewanks Function without Bounds(F7) & {$[0,600]^{D}$} & -180 \\
$f_{8}$ & Shifted Rotated Ackley's Function with Global Optimum on & {$[-32,32]^{D}$} & -140 \\
& Bounds(F8) & {$[-5,5]^{D}$} & -330 \\
$f_{9}$ & Shifted Rastrigins Function(F9) & {$[-5,5]^{D}$} & -330 \\
$f_{10}$ & Shifted Rotated Rastrigin's Function(F10) & {$[-0.5,0.5]^{D}$} & 90 \\
$f_{11}$ & Shifted Rotated Weierstrass Function(F11) & {$[-\pi, \pi]^{D}$} & -460 \\
$f_{12}$ & Schwefels Problem 2.13(F12) & {$[-5,5]^{D}$} & -130 \\
$f_{13}$ & Expanded Extended Griewank's plus Rosenbrock's Func- & & \\
& tion(F13) & {$[-100,100]^{D}$} & -300 \\
$f_{14}$ & Shifted Rotated Expanded Scaffer's F6(F14) & {$[-5,5]^{D}$} & 120 \\
$f_{15}$ & Hybrid Composition Function(F15) &
\end{tabular}

F-Function D-Dimensionality of the function S-The ranges of variables $f_{\min }$ - Minimum value of the function

Table 4: Comparison results of the best value, the median, the worst value, average, and standard deviation of the functions $\mathrm{g}_{1}$ to $\mathrm{g}_{15}$ for 50 independent runs using CGA,CEA and GA methods

\begin{tabular}{|c|c|c|c|c|c|c|c|c|c|}
\hline 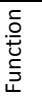 & 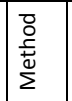 & 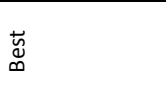 & 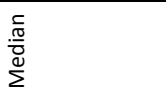 & $\begin{array}{l}\text { th } \\
\text { 帘 }\end{array}$ & 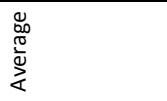 & i & $\mathrm{x}_{1}$ & $x_{2}$ & 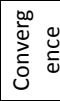 \\
\hline & $\mathrm{CGA}$ & $1.1134 \mathrm{e}-10$ & $9.9101 \mathrm{e}-10$ & $4.0103 e-09$ & $1.4509 \mathrm{e}-09$ & $1.0264 \mathrm{e}-09$ & $-0.9543 e-05$ & $0.4502 e-05$ & 48 \\
\hline g1 & CEA & $3.1897 e-11$ & $1.9406 \mathrm{e}-10$ & $2.6118 \mathrm{e}-10$ & $1.9574 \mathrm{e}-10$ & $7.0675 e-11$ & $0.4807 e-05$ & $-0.2965 e-05$ & 17 \\
\hline & GA & $4.6568 e-06$ & $4.6568 e-06$ & $2.3284 e-05$ & $1.3970 e-05$ & $9.4081 e-06$ & 0.0015 & 0.0015 & 136 \\
\hline \multirow{3}{*}{ g2 } & $\mathrm{CGA}$ & $4.2230 \mathrm{e}-10$ & $4.2230 \mathrm{e}-10$ & $4.2230 \mathrm{e}-10$ & $4.2230 \mathrm{e}-10$ & $3.6557 e-25$ & 1.0000 & 1.0000 & 95 \\
\hline & CEA & $2.3516 \mathrm{e}-08$ & $2.3516 \mathrm{e}-08$ & $2.0979 \mathrm{e}-07$ & $4.3060 \mathrm{e}-08$ & $5.5168 e-08$ & 1.0000 & 1.0000 & 15 \\
\hline & GA & $7.2552 e-07$ & $1.8174 \mathrm{e}-05$ & $5.1102 e-05$ & $2.2489 e-05$ & $1.5667 e-05$ & 1.0008 & 1.0017 & 172 \\
\hline \multirow{3}{*}{ g3 } & CGA & $4.1684 \mathrm{e}-05$ & $2.2875 e-04$ & $3.7598 \mathrm{e}-04$ & $2.2850 \mathrm{e}-04$ & $8.3228 e-05$ & $-0.0491 e-04$ & $-0.1389 e-04$ & 111 \\
\hline & CEA & $4.3708 e-05$ & $4.6823 e-04$ & $6.9224 \mathrm{e}-04$ & $4.3041 \mathrm{e}-04$ & $2.0039 \mathrm{e}-04$ & $-0.0623 e-04$ & $-0.1414 \mathrm{e}-04$ & 15 \\
\hline & $\mathrm{GA}$ & 0.0020 & 0.0020 & 0.0034 & 0.0023 & $8.6401 \mathrm{e}-04$ & $-0.4883 e-03$ & $-0.4883 e-03$ & 62 \\
\hline \multirow{3}{*}{ g4 } & CGA & $7.7170 e-10$ & $1.3390 \mathrm{e}-08$ & $3.2317 e-08$ & $1.6150 \mathrm{e}-08$ & $8.6917 e-09$ & $0.1915 e-05$ & $0.0473 e-05$ & 57 \\
\hline & CEA & $1.0093 e-10$ & $1.8868 \mathrm{e}-07$ & $7.1941 \mathrm{e}-07$ & $2.6063 e-07$ & $2.2747 \mathrm{e}-07$ & $0.6054 e-06$ & $-0.3771 e-06$ & 18 \\
\hline & GA & $2.4219 e-06$ & $1.2109 \mathrm{e}-05$ & $2.1797 e-05$ & $1.1722 e-05$ & $7.0457 e-06$ & $0.7813 e-04$ & $-0.7813 e-04$ & 85 \\
\hline \multirow{3}{*}{ g5 } & CGA & 0.0050 & 0.0050 & 0.0050 & 0.0050 & $1.6831 \mathrm{e}-06$ & $0.3011 e-03$ & $-0.6374 \mathrm{e}-03$ & 68 \\
\hline & $\mathrm{CEA}$ & 0.0050 & 0.0050 & 0.0051 & 0.0050 & $2.2805 e-05$ & $0.1725 e-03$ & $0.7287 e-03$ & 18 \\
\hline & GA & 0.0061 & 0.0127 & 0.0143 & 0.0121 & 0.0023 & 0.0092 & 0.0641 & 110 \\
\hline \multirow{3}{*}{ g6 } & CGA & $1.5148 e-10$ & $8.9784 e-09$ & $1.2233 e-08$ & $9.1321 \mathrm{e}-09$ & $5.3891 \mathrm{e}-09$ & 0. $1227 \mathrm{e}-04$ & $-0.1322 e-04$ & 85 \\
\hline & CEA & $5.5333 e-10$ & $3.2741 \mathrm{e}-08$ & $9.7317 e-08$ & $5.4932 e-08$ & $4.1444 \mathrm{e}-08$ & $0.2345 e-04$ & $-0.2161 e-04$ & 21 \\
\hline & $\mathrm{GA}$ & $1.0000 e-06$ & $1.3000 e-05$ & $2.5001 \mathrm{e}-05$ & $1.4360 e-05$ & $7.0735 e-06$ & -0.0010 & 0.0010 & 89 \\
\hline \multirow{3}{*}{ g7 } & CGA & $1.7325 e-05$ & $1.5898 \mathrm{e}-04$ & $2.6459 \mathrm{e}-04$ & $1.6789 \mathrm{e}-04$ & $6.6164 \mathrm{e}-05$ & $-0.1733 e-04$ & -0.1180 e -04 & 36 \\
\hline & CEA & $5.4528 e-05$ & $2.2733 e-04$ & 0.0010 & $4.5618 \mathrm{e}-04$ & $3.1448 \mathrm{e}-04$ & $-0.5453 e-04$ & $0.0392 \mathrm{e}-04$ & 20 \\
\hline & GA & 0.0015 & 0.0046 & 0.0076 & 0.0052 & 0.0022 & -0.0015 & 0.0015 & 49 \\
\hline \multirow{4}{*}{ g8 } & CGA & $3.8332 e-06$ & $2.4977 e-05$ & $5.5813 e-05$ & $2.8145 e-05$ & $1.4871 \mathrm{e}-05$ & $-0.3015 e-05$ & $0.0818 e-05$ & 77 \\
\hline & CEA & $7.7840 e-06$ & $4.5508 e-05$ & $5.1125 e-05$ & $3.7532 e-05$ & $1.0428 e-05$ & $0.5559 e-05$ & $-0.2225 e-05$ & 15 \\
\hline & GA & $3.0520 e-04$ & $9.1566 \mathrm{e}-04$ & $9.1575 e-04$ & $7.5087 e-04$ & $2.4052 e-04$ & $0.1526 \mathrm{e}-03$ & $0.1526 e-03$ & 142 \\
\hline & CGA & 0 & 0 & 0 & 0 & 0 & -0.0839 & -0.3983 & 6 \\
\hline
\end{tabular}




\begin{tabular}{|c|c|c|c|c|c|c|c|c|c|}
\hline \multirow[t]{2}{*}{ g9 } & CEA & 0 & 0 & 0 & 0 & 0 & -0.1968 & -0.2823 & 6 \\
\hline & GA & 0 & 0 & 0 & 0 & 0 & 0.0839 & 0.4288 & 5 \\
\hline \multirow{3}{*}{ g10 } & CGA & $1.3366 e-41$ & $1.3366 e-41$ & $3.9463 e-37$ & $1.7375 e-37$ & $1.9778 e-37$ & $-0.7630 e-04$ & $-0.7630 e-04$ & 11 \\
\hline & CEA & $4.8118 e-45$ & $1.6943 e-41$ & $1.6943 e-41$ & $1.6200 e-41$ & $3.3577 e-42$ & -0.0091 & -0.0015 & 15 \\
\hline & $\mathrm{GA}$ & $5.2160 e-29$ & $7.6683 e-22$ & $2.2810 e-20$ & $3.8371 e-21$ & $5.8580 e-21$ & -0.0009 & -0.0015 & 10 \\
\hline \multirow{3}{*}{ g11 } & CGA & -1.0000 & -1.0000 & -1.0000 & -1.0000 & $7.8505 e-16$ & 3.1418 & 3.1418 & 27 \\
\hline & CEA & -1.0000 & -0.9999 & -0.9999 & -0.9999 & $8.6618 e-06$ & 3.1418 & 3.1418 & 16 \\
\hline & $\mathrm{GA}$ & -1.0000 & -0.9995 & -0.9986 & -0.9995 & $3.5878 e-04$ & 3.1418 & 3.1418 & 19 \\
\hline \multirow{3}{*}{ g12 } & CGA & 0.9980 & 0.9980 & 0.9980 & 0.9980 & $6.5252 e-14$ & -31.9848 & -31.9829 & 93 \\
\hline & CEA & 0.9980 & 0.9980 & 0.9980 & 0.9980 & $3.6126 e-11$ & -31.9778 & -31.9798 & 20 \\
\hline & GA & 0.9980 & 0.9980 & 0.9980 & 0.9980 & $1.9323 e-10$ & -31.9775 & -31.9775 & 99 \\
\hline \multirow{3}{*}{ g13 } & $\mathrm{CGA}$ & 3.0000 & 3.0000 & 3.0000 & 3.0000 & $3.1201 e-09$ & 0.0000 & -1.0000 & 65 \\
\hline & $\mathrm{CEA}$ & 3.0000 & 3.0000 & 3.0000 & 3.0000 & $4.1948 e-08$ & 0.0000 & -1.0000 & 21 \\
\hline & $\mathrm{GA}$ & 3.0000 & 3.0000 & 3.0000 & 3.0000 & $1.2456 e-05$ & 0.0000 & -1.0000 & 114 \\
\hline \multirow{3}{*}{ g14 } & CGA & -1.0316 & -1.0316 & -1.0316 & -1.0316 & $4.7386 e-06$ & 0.0896 & -0.7125 & 43 \\
\hline & $\mathrm{CEA}$ & -1.0316 & -1.0316 & -1.0316 & -1.0316 & $9.9630 e-06$ & -0.0897 & 0.7131 & 24 \\
\hline & $\mathrm{GA}$ & -1.0316 & -1.0316 & -1.0316 & -1.0316 & $1.3930 e-05$ & -0.0897 & 0.7184 & 48 \\
\hline \multirow{3}{*}{ g15 } & CGA & 0.3979 & 0.3979 & 0.3979 & 0.3979 & $1.3339 e-08$ & 9.4248 & 2.4749 & 112 \\
\hline & $\mathrm{CEA}$ & 0.3979 & 0.3979 & 0.3979 & 0.3979 & $1.1516 e-06$ & -3.1416 & 12.2751 & 21 \\
\hline & $\mathrm{GA}$ & 0.3979 & 0.3979 & 0.3979 & 0.3979 & $1.9852 e-06$ & 9.4250 & 2.4752 & 90 \\
\hline
\end{tabular}

Table 5: Comparison results of the best value, the median, the worst value, average, and standard deviation of the functions $f_{1}$ to $f_{15}$ for 50 independent runs using CGA,CEA and GA methods

\begin{tabular}{|c|c|c|c|c|c|c|c|c|c|}
\hline$\Xi$ 㑒 & 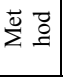 & 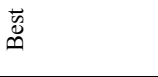 & $\sum^{\bar{e}} . \pm$ & $\vec{b}^{0}$ & $\stackrel{8}{\rightleftarrows}$ & $\stackrel{\theta}{\dot{s}}$ & $\mathrm{x}_{1}$ & $\mathrm{x}_{2}$ & పี \\
\hline \multirow{3}{*}{$\mathrm{fl}$} & CGA & -449.9998 & -449.9901 & -449.9901 & -449.9924 & 0.0030 & -39.3068 & 58.8855 & 45 \\
\hline & CEA & -449.9989 & -449.9565 & -449.9229 & -449.9578 & 0.0244 & -39.2782 & 58.9014 & 10 \\
\hline & GA & -449.9745 & -449.9120 & -449.7904 & -449.8864 & 0.0498 & -39.2447 & 59.0448 & 1000 \\
\hline \multirow{3}{*}{$\mathrm{f} 2$} & CGA & -450.0000 & -450.0000 & -449.9987 & -449.9998 & $4.1997 e-04$ & 35.6202 & -82.9080 & 67 \\
\hline & CEA & -449.9942 & -449.8769 & -449.8063 & -449.8830 & 0.0558 & 35.6727 & -82.8978 & 10 \\
\hline & $\mathrm{GA}$ & -449.9964 & -449.9494 & -449.9454 & -449.9586 & 0.0189 & 35.6588 & -82.9953 & 998 \\
\hline \multirow{3}{*}{ f3 } & CGA & -449.9942 & -449.9668 & -449.9668 & -449.9692 & 0.0058 & -32.2347 & 64.9836 & 42 \\
\hline & CEA & -447.0247 & -446.9247 & -446.2247 & -446.7718 & 0.3217 & -30.5043 & 64.6693 & 10 \\
\hline & GA & -449.9895 & -449.8582 & -449.8582 & -449.8749 & 0.0441 & -32.2809 & 64.9920 & 77 \\
\hline \multirow{3}{*}{$\mathrm{f} 4$} & CGA & -449.9999 & -449.9943 & -449.6849 & -449.9821 & 0.0012 & 35.6344 & -82.9252 & 1000 \\
\hline & CEA & -449.9958 & -449.9040 & -449.7769 & -449.8975 & 0.0689 & 35.6161 & -82.9465 & 10 \\
\hline & GA & -449.9997 & -449.9865 & -449.9657 & -449.9853 & 0.0100 & 35.6169 & -82.9126 & 1000 \\
\hline
\end{tabular}




\begin{tabular}{|c|c|c|c|c|c|c|c|c|c|}
\hline \multirow{3}{*}{ f5 } & CGA & -310 & -310 & -310 & -310 & 0 & 100 & 100 & 20 \\
\hline & CEA & -310 & -310 & -310 & -310 & 0 & 100 & 100 & 4 \\
\hline & GA & -309.8292 & -309.0295 & -308.4863 & -309.0329 & 0.3597 & 100.0000 & 99.9939 & 123 \\
\hline \multirow{3}{*}{ f6 } & CGA & 390.0000 & 390.0001 & 390.0004 & 390.0002 & $9.7598 e-05$ & 81.0241 & -48.3932 & 67 \\
\hline & CEA & 390.0000 & 390.0125 & 390.0049 & 390.0058 & 0.0037 & 81.0254 & -48.3912 & 18 \\
\hline & $\mathrm{GA}$ & 390.0001 & 390.1423 & 390.3480 & 390.1501 & 0.0963 & 81.0176 & -48.4058 & 95 \\
\hline \multirow{3}{*}{ f7 } & CGA & -179.9919 & -179.6063 & -178.2872 & -179.8255 & 0.3884 & -271.9132 & -13.3285 & 54 \\
\hline & CEA & -179.9866 & -179.6100 & -179.1093 & -179.6018 & 0.2142 & -271.8144 & -13.3803 & 10 \\
\hline & GA & -140.7319 & -139.7547 & -139.6343 & -139.8996 & 0.3557 & 1.9318 & 0.1282 & 258 \\
\hline \multirow{3}{*}{ f8 } & CGA & -139.8350 & -136.4446 & -135.7379 & -136.7568 & 1.1469 & -31.9936 & 15.0032 & 3 \\
\hline & CEA & -139.6152 & -135.4985 & -134.6663 & -135.9566 & 1.2842 & -31.9924 & 15.0095 & 3 \\
\hline & GA & -137.3716 & -131.0882 & -129.1634 & -131.5795 & 2.1122 & -31.8037 & 15.7205 & 3 \\
\hline \multirow{3}{*}{ f9 } & CGA & -329.9997 & -329.9539 & -329.9056 & -329.9552 & 0.0281 & 1.9017 & -1.5644 & 3 \\
\hline & CEA & -329.9977 & -329.8700 & -329.7644 & -329.8822 & 0.0715 & 1.8994 & -1.5612 & 3 \\
\hline & GA & -329.9164 & -329.0696 & -328.8314 & -329.2013 & 0.3453 & 1.9035 & -1.5847 & 3 \\
\hline \multirow{3}{*}{ f10 } & CGA & -329.9988 & -329.7047 & -329.5676 & -329.8058 & 0.0836 & 1.9018 & -1.5626 & 56 \\
\hline & CEA & -329.9964 & -329.7202 & -329.5477 & -329.7277 & 0.1476 & 1.9030 & -1.5626 & 8 \\
\hline & $\mathrm{GA}$ & -329.9728 & -328.8565 & -328.4160 & -328.9917 & 0.4484 & 1.8939 & -1.5664 & 547 \\
\hline \multirow{3}{*}{ f11 } & CGA & 90.0025 & 90.0230 & 90.0261 & 90.0214 & 0.0056 & -0.1367 & 0.1186 & 273 \\
\hline & CEA & 90.0400 & 90.0246 & 90.0280 & 90.0230 & 0.0044 & -0.1365 & 0.1186 & 18 \\
\hline & GA & 90.0400 & 90.0924 & 90.1021 & 90.0911 & 0.0129 & -0.1365 & 0.1186 & 390 \\
\hline \multirow{3}{*}{$\mathrm{f} 12$} & CGA & -459.9998 & -459.9750 & -459.9479 & -459.9743 & 0.0145 & -1.1443 & -1.9036 & 34 \\
\hline & CEA & -459.9988 & -459.9921 & -459.9905 & -459.9930 & 0.0024 & -1.1442 & -1.9037 & 16 \\
\hline & $\mathrm{GA}$ & -459.9990 & -459.9547 & -459.9503 & -459.9638 & 0.0159 & -1.1451 & -1.9033 & 64 \\
\hline \multirow{3}{*}{ f13 } & CGA & -130.0000 & -130.0000 & -130.0000 & -130.0000 & $1.8583 e-05$ & 0.2466 & -0.8516 & 72 \\
\hline & CEA & -130.0000 & -129.9994 & -129.9942 & -129.9986 & 0.0016 & 0.2497 & -0.8462 & 10 \\
\hline & GA & -130.0000 & -129.9999 & -129.9999 & -129.9999 & $2.7233 e-05$ & 0.2488 & -0.8465 & 165 \\
\hline
\end{tabular}




\begin{tabular}{|c|c|c|c|c|c|c|c|c|c|}
\hline \multirow{3}{*}{ f14 } & CGA & -299.9902 & -299.9805 & -299.9804 & -299.9807 & 0.0014 & -73.5714 & -23.5492 & 3 \\
\hline & CEA & -299.9806 & -299.9518 & -299.9252 & -299.9518 & 0.0217 & -74.3038 & -24.3275 & 3 \\
\hline & GA & -299.9805 & -299.9528 & -299.9236 & -299.9514 & 0.0226 & -71.2337 & -25.2216 & 3 \\
\hline \multirow{3}{*}{ f15 } & CGA & 120.0001 & 120.0014 & 120.0076 & 120.0017 & 0.0015 & 3.3252 & -1.2834 & 58 \\
\hline & CEA & 120.0008 & 120.0014 & 120.0018 & 120.0015 & 0.1309 & 3.3252 & -1.2832 & 10 \\
\hline & GA & 120.0002 & 120.0018 & 120.0018 & 120.0016 & $4.4508 e-04$ & 3.3253 & -1.2837 & 111 \\
\hline
\end{tabular}

Table 6: Comparison results - The number of generations for convergence of the functions $\mathrm{g}_{1}$ to $\mathrm{g}_{15}$ using CGA,CEA and GA methods

\begin{tabular}{|c|c|c|c|c|c|c|c|c|c|c|c|c|c|c|c|c|}
\hline $\mathrm{F}$ & & $g_{1}$ & $g_{2}$ & $g_{3}$ & $g_{4}$ & $g_{5}$ & $g_{6}$ & $g_{7}$ & $g_{8}$ & $g_{9}$ & $g_{10}$ & $g_{11}$ & $g_{12}$ & $g_{13}$ & $g_{14}$ & $g_{15}$ \\
\hline \multirow{3}{*}{ Method } & CGA & 48 & 95 & 111 & 57 & 68 & 85 & 36 & 77 & 6 & 11 & 27 & 93 & 65 & 43 & 112 \\
\hline & CEA & 17 & 15 & 15 & 18 & 18 & 21 & 20 & 15 & 6 & 15 & 16 & 20 & 21 & 24 & 21 \\
\hline & $\mathrm{GA}$ & 136 & 172 & 62 & 85 & 110 & 89 & 49 & 142 & 5 & 10 & 19 & 99 & 114 & 48 & 90 \\
\hline
\end{tabular}

Table 7: Comparison results - The number of generations for convergence of the functions $\mathrm{f}_{1}$ to $\mathrm{f}_{15}$ using CGA,CEA and GA methods

\begin{tabular}{|c|c|c|c|c|c|c|c|c|c|c|c|c|c|c|c|c|}
\hline $\mathrm{F}$ & & $f_{1}$ & $f_{2}$ & $f_{3}$ & $f_{4}$ & $f_{5}$ & $f_{6}$ & $f_{7}$ & $f_{8}$ & $f_{9}$ & $f_{10}$ & $f_{11}$ & $f_{12}$ & $f_{13}$ & $f_{14}$ & $f_{15}$ \\
\hline \multirow{3}{*}{ Method } & $\overline{\mathrm{CGA}}$ & 45 & 67 & 42 & 1000 & 20 & 67 & 54 & 3 & 3 & 56 & 273 & 34 & 72 & 3 & 58 \\
\hline & CEA & 10 & 10 & 10 & 10 & 4 & 18 & 10 & 3 & 3 & 8 & 18 & 16 & 10 & 3 & 10 \\
\hline & GA & 1000 & $\begin{array}{l}998 \\
\end{array}$ & 77 & 1000 & 123 & 95 & 258 & 3 & 3 & 547 & 390 & 64 & 165 & 3 & 111 \\
\hline
\end{tabular}

F-Function

\section{CONCLUSION}

In this paper we have introduced a new operator for genetic algorithm which imitates the biological cloning process and have applied the integrated algorithm on a number of standard test cases. We have simulated the results for the standard GA as well as two new optimization methods obtained by integrating the new clone operator with the GA operators. The results of the three methods, namely proposed CGA method, proposed CEA method and standard GA, indicate that the former is capable of generating better solution quality than the other methods. It is seen that the CGA and CEA methods significantly improve the convergence rate and the quality of the solution. In conclusion, it can be said that the proposed operator reduces the number of searches within the solution space and enhances the convergence capability and the performance of the GA. The proposed method can also be analyzed using real coded GAs with specially designed operators and also be extended to higher dimensions. This will be considered in future work. 


\section{ACKNOWLEDGEMENTS}

The authors would like to thank Abhay Kumar, Group Director AFDG, S.Pandian, Deputy Director AERO and K.Sivan, Director VSSC for encouragement and support and also for providing the computational facilities.

\section{REFERENCES}

[1] J. H. Holland, Adaptation in natural and artificial systems: An introductory analysis with applications to biology, control, and artificial intelligence. U Michigan Press, 1975.

[2] L. Davis, "Genetic algorithms and simulated annealing," 1987.

[3] D. Goldberg, Genetic Algorithms in Search, Optimization, and Machine Learning, Artificial Intelligence. Addison-Wesley Publishing Company, 1989.

[4] K. Man, K. Tang, and S. Kwong, "Genetic algorithms: Concepts and designs", Springer, 1999.

[5] D. Beasley, R. Martin, and D. Bull, "An overview of genetic algorithms: Part 1. fundamentals," University computing, vol. 15, pp. 58-58, 1993

[6] L. Davis, Handbook of genetic algorithms. New York: Van Nostrand Reinhold, 1991.

[7] J. J. Grefenstette, "Optimization of control parameters for genetic algorithms," Systems, Man and Cybernetics, IEEE Transactions on, vol. 16, no. 1, pp. 122-128, 1986.

[8] T. Back, "Evolutionary algorithms in theory and practice," 1996.

[9] Z. Michalewicz, Genetic algorithms + data structures $=$ evolution programs. Springer Science \& Business Media, 2013.

[10] K. De Jong, "Genetic algorithms: a 30 year perspective," Perspectives on Adaptation in Natural and Artificial Systems, vol. 11, 2005.

[11] J. E. Baker, "Adaptive selection methods for genetic algorithms," in Proceedings of an International Conference on Genetic Algorithms and their applications. Hillsdale, New Jersey, 1985, pp. 101-111.

[12] Brindle and U. of Alberta. Department of Computing Science, Genetic Algorithms for Function Optimization, ser. University of Alberta Department of Computing Science Technical Report Tr. Thesis (Ph.D.) - University of Alberta, 1981.

[13] D. Whitley, "Using reproductive evaluation to improve genetic search and heuristic discovery," in Genetic algorithms and their applications: proceedings of the second International Conference on Genetic Algorithms: July 28-31, 1987 at the Massachusetts Institute of Technology, Cambridge, MA. Hillsdale, NJ: L. Erlhaum Associates, 1987. 
[14] J. E. Baker, "Reducing bias and inefficiency in the selection algorithm,"in Proceedings of the second international conference on genetic algorithms, 1987, pp. 14-21.

[15] L. D. Whitley et al., "The genitor algorithm and selection pressure: Why rankbased allocation of reproductive trials is best." in ICGA, vol. 89, 1989, pp. $116-123$.

[16] G. Sywerda, "Uniform crossover in genetic algorithms," in Proceedings of the third international conference on Genetic algorithms. Morgan Kaufmann Publishers Inc., 1989, pp. 2-9.

[17] S. M. Elsayed, R. A. Sarker, and D. L. Essam, "A new genetic algorithm for solving optimization problems," Engineering Applications of Artificial Intelligence, vol. 27, pp. 57-69, 2014.

[18] H. M. Pandey, A. Chaudhary, and D. Mehrotra, "A comparative review of approaches to prevent premature convergence in ga," Applied Soft Computing, vol. 24, pp. 1047-1077, 2014.

[19] D. E. Goldberg and K. Deb, "A comparative analysis of selection schemes used in genetic algorithms," Foundations of genetic algorithms, vol. 1, pp. 6993, 1991.

[20] F. Liu and G. Zeng, "Study of genetic algorithm with reinforcement learning to solve the tsp," Expert Systems with Applications, vol. 36, no. 3, pp. 6995$7001,2009$.

[21] M. Thakur, S. S. Meghwani, and H. Jalota, "A modified real coded genetic algorithm for constrained optimization," Applied Mathematics and Computation, vol. 235, pp. 292-317, 2014.

[22] K. DeJong, The Analysis and behaviour of a Class of Genetic Adaptive Systems. Thesis (Ph.D.)-University of Michigan, 1975.

[23] J. Chen, S.-y. Zhang, Z. Gao, and L.-X. Yang, "Feature-based initial population generation for the optimization of job shop problems," Journal of Zhejiang University SCIENCE C, vol. 11, no. 10, pp. 767-777, 2010.

[24] V. To gan and A. T. Dalo glu, "An improved genetic algorithm with initial population strategy and self-adaptive member grouping," Computers \& Structures, vol. 86, no. 11, pp. 1204-1218, 2008.

[25] D. Sharma, K. Deb, and N. Kishore, "Domain-specific initial population strategy for compliant mechanisms using customized genetic algorithm," Structural and Multidisciplinary Optimization, vol. 43, no. 4, pp. 541-554, 2011.

[26] Z. H. Ahmed, "The ordered clustered travelling salesman problem: a hybrid genetic algorithm," The Scientific World Journal, vol. 2014, 2014.

[27] D. Goldberg and R. Lingle, "Alleles, loci and the tsp en: Proceedings of the first international conference on genetic algorithms," 1985. 
[28] K. D. Jong, “Adaptive system design: a genetic approach," Systems, Man and Cybernetics, IEEE Transactions on, vol. 10, no. 9, pp. 566-574, 1980.

[29] D. E. Goldberg and M. P. Samtani, "Engineering optimization via genetic algorithm," in Electronic Computation (1986). ASCE, 1986, pp. 471- 482.

[30] D. E. Goldberg and C. H. Kuo, "Genetic algorithms in pipeline optimization," Journal of Computing in Civil Engineering, vol. 1, no. 2, pp. 128-141, 1987. 